necessarily describe organic materials using the same models that have been developed for metals and inorganic semiconductors ${ }^{9}$ because charges in organics propagate in very narrow bands, created by the overlap of the highest occupied and lowest unoccupied molecular orbitals (HOMO and LUMO) of the weakly interacting molecules. Typically, there is only one type of carrier - electron or hole - so spin-flipping mechanisms resulting from an interaction between the two can be ruled out. The hyperfine interaction is also expected to be weak as most of the charge density associated with the HOMO and the LUMO is concentrated around the carbon atoms and in its most abundant isotopic form, ${ }^{12} \mathrm{C}$, carbon does not possess a nuclear spin. Finally, the strength of the spin-orbit interaction scales as the fourth power of the atomic number. In organics, which are made of light atoms, this interaction will be relatively weak compared with 'heavier' semiconductors.

Bandyopadhyay and colleagues compare their data for laterally confined spin valves with extended structures, such as thin films, that are published in the literature ${ }^{6,7}$. Based on a number of arguments, they conclude that the socalled 'Elliott-Yafet' mechanism, which depends on the spin-orbit interaction, dominates the spin-diffusion. This is the third major contribution from their work and is indeed an interesting suggestion. However, does this work solve all the puzzles? The answer, certainly, is no.

One problem is that measurements of the spin relaxation time are largely limited to transport measurements as the optical probes that have worked so well for semiconductors are not useful in organics. This is a direct consequence of the weak spin-orbit interaction. So far the spin-diffusion length in organic spin valves has always been determined from the Jullière formula ${ }^{10}$, which relates the measured magnetoresistance to the polarization of the spins in the magnetic electrodes and the spin-diffusion length. However, the details of the spin-filtering properties of the organic-magnetic metal interface must really be understood in greater detail. For instance, Bandyopadhyay's group assumes that the spin-polarization at the $\mathrm{Co}$ and $\mathrm{Ni}$ electrodes is determined entirely by the $d$ electrons in these materials, but $s$ electrons may in fact make a substantial contribution.

More importantly, the dominant mechanism for spin-diffusion in organics may be completely different from that in standard metals and semiconductors. Molecular layers are rich in unpaired spins, originating from dangling bonds, triplet states and free radicals.

These can act as strong spin-flip centres, completely undoing the benefit of the weak spin-orbit coupling. Independent measurements of the spin-flip centre densities and electron mobility are paramount, though these measurements are certain to be more difficult in nanostructures, compared with the bulk.

Spin transport in organics is a fascinating new area, where the convergence of chemistry, physics, electronic engineering and eventually biology can open new and unexpected avenues. Fully characterized systems are needed, and so this is the first challenge for the future of organic spintronics.

\footnotetext{
References

1. Bass, J. \& Pratt, W. P. Jr. Preprint at <http://arxiv.org/condmat/0610085>

2. Kikkawa, J. \& Awschalom, D. D. Nature 397, 139-141 (1999).

3. Pramanik, S. et al. Nature Nanotech. 2, 216-219 (2007).

4. Baibich, M. N. et al. Phys. Rev. Lett. 61, 2472-2475 (1988).

5. Binasch, G. et al. Phys. Rev. B 39, 4828-4830 (1989).

6. Xiong, Z. H., Wu, D., Valy Vardeny Z. \& Shi, J. Nature $\mathbf{4 2 7}$ 821-824 (2004).

7. Riminucci, A. et al. Preprint at $<$ http://arxiv.org/condmat/0701603>

8. Tsukagoshi, K., Alphenaar B. W. \& Ago, H. Nature 401, 572-574 (1999).

9. Sanvito S. \& Rocha, A. R. J. Comput. Theor. Nanos. 3 , 624-642 (2006).

10. Jullière, M. Phys. Lett. A 54, 225-226 (1975).
}

\title{
MAGNETISM
}

\section{Vortex ups and downs}

In a ferromagnetic nanodisk, the magnetization tends to swirl around in the plane of the disk and can point either up or down at the centre of this 'magnetic vortex'. This binary state could be useful for information storage. However, forcing the vortex core to flip from up to down can require a fairly large magnetic field or long switching times. Now, two separate teams look at different approaches to overcome these technological hurdles.

In a combination of experimental and theoretical work, Teruo Ono of Kyoto University and colleagues in Japan and France, show that an oscillating current in which the electron spins are polarized in a certain direction can switch the vortex core in a permalloy nanodisk (Nature Mater. 6, 269-273; 2007). The spin current exerts a torque on the vortex core, which, according to simulation, causes it to
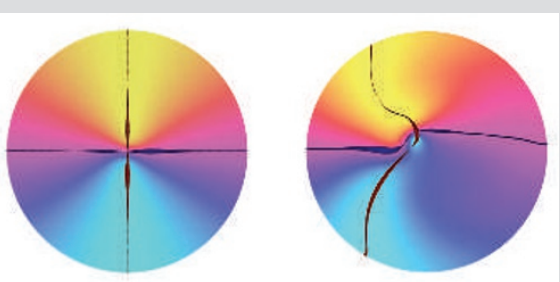

short magnetic field pulse is applied in the plane of the disk (Phys. Rev. Lett. 98, 117201; 2007). They find that a short pulse of about 60 picoseconds and 800 Gauss in strength (the earth's magnetic field is 0.5 Gauss) is enough to switch the vortex direction. Vortices disappear and are reformed where the direction of the in-plane magnetization changes sharply. The image is a simulation by the German group, which shows, starting at the top left, how a vortex (the point at the centre where the different colours meet) can be erased by a magnetic pulse and then reform elsewhere.

Taken together, the studies show that short magnetic field pulses provide faster switching, but electrical vortex switching may be more technologically viable. Bit by bit, however, magnetic vortices are becoming more promising candidates for information storage.

Jessica Thomas 